\title{
Controlled NO-Release from 3D-Printed Small-Diameter Vascular Grafts Prevents Platelet Activation and Bacterial Infectivity
}

\author{
Fatemeh Kabirian, ${ }^{\dagger, \dagger}$ Bartosz Ditkowski, ${ }^{\dagger}$ Ali Zamanian, ${ }^{\dagger}$ Marc F. Hoylaerts, $^{\S}$ Masoud Mozafari, ${ }^{*}, \dagger, \|, \perp_{\odot}$ \\ and Ruth Heying

\begin{abstract}
${ }^{\dagger}$ Nanotechnology and Advanced Materials Department, Materials and Energy Research Center (MERC), P.O. Box 14155-4777, Tehran, Iran

"Cardiovascular Developmental Biology, Department of Cardiovascular Sciences, KU Leuven, 3000 Leuven, Belgium

${ }^{\S}$ Center of Molecular and Vascular Biology, Department of Cardiovascular Sciences, KU Leuven, 3000 Leuven, Belgium

"Cellular and Molecular Research Center, Iran University of Medical Sciences (IUMS), Tehran, 1449614535, Iran

${ }^{\perp}$ Department of Tissue Engineering \& Regenerative Medicine, Faculty of Advanced Technologies in Medicine, Iran University of
\end{abstract} \\ Medical Sciences (IUMS), Tehran, 1449614535, Iran
}

Supporting Information

\begin{abstract}
Thrombogenicity and bacterial infectiveness are the most common complications for foreign blood contacting surfaces associated with functional failure of small-diameter vascular grafts (SDVGs). In this work, novel bactericidal and nonthrombogenic SDVGs were manufactured via 3Dprinting technology, thus producing a controlled nitric oxide (NO) release coating. S-Nitroso- $N$-acetyl-D-penicillamine (SNAP) was synthesized as an NO-donor, and three biomedical grade composite matrixes of poly(ethylene glycol) (PEG)-SNAP, polycaprolactone (PCL)-SNAP, and PEG-PCLSNAP were validated for water uptake and NO-release kinetics. To optimize and extend the NO releasing profile, a PCL top-coat (tc) was deposited over the NO-releasing layer. The PEG-PCL-SNAP-tc was selected for biological tests as its NO-release profile was prolonged and well-controlled. Coating the 3D-printed SDVG with PEG-PCL-SNAP-tc

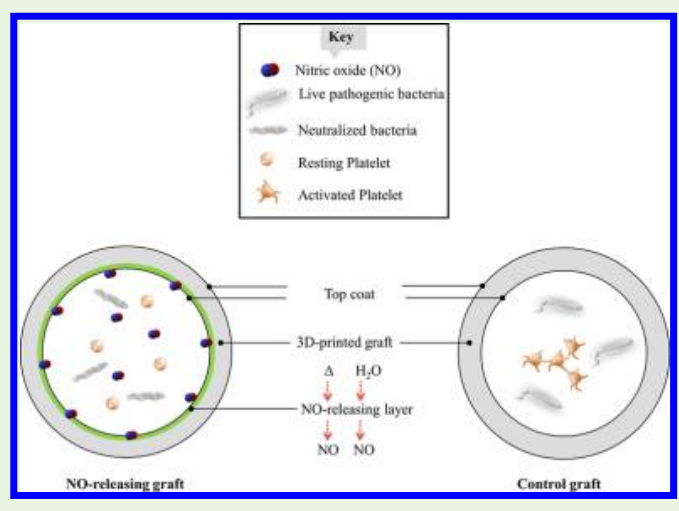
resulted in quantitative antibacterial features against both Gram-positive and Gram-negative bacteria and in NO-mediated inhibition of platelet activation and aggregation. Antibacterial and antithrombogenic properties in plasma are expected to be as effective as in PBS, since NO release in plasma was not significantly different from that in PBS. Overall, application of the inexpensive, rapid, and reproducible 3D-printing technology as a custom-based production method, in combination with a well-controlled NO release system, is promising for the production of innovative bactericidal and hemocompatible SDVGs.
\end{abstract}

KEYWORDS: 3D-printing, small-diameter vascular grafts, nitric oxide, controlled release, antibacterial, hemocompatibility

\section{INTRODUCTION}

Approximately 1.4 million patients per year require vascular prostheses in the USA. ${ }^{1}$ Autologous vessels, which are the gold standard for bypass surgery, have limited availability due to the advanced age of patients, previous harvesting, or pre-existing arterial disease. ${ }^{2}$ Therefore, synthetic vascular grafts are needed to overcome deficient autograft availability. Various methods such as electrospinning, freeze-drying, dip coating, and phase separation have been applied for vascular graft fabrication. The drawback of these methods is the small control over the structure and morphology of the fabricated object. ${ }^{3}$ In addition, to address the more complex vascular network architecture and its intricate details, such as bifurcations or curvatures and the individual defect anatomy of each patient, which is specific, an accurate design and fabrication method such as three-dimensional (3D) printing seems preferable. ${ }^{4-7}$ Nowadays, 3D printing becomes an inexpensive and rapid manufacturing method, which can produce reproducible patient-specific medical implants in small numbers, with minimum material waste and precise control over spatial complexity of the constructs and with tunable mechanical and biological properties. ${ }^{8}$ 3D-printers produce high-resolution biodegradable objects through the deposition of sequential layers based on 3D computer-aided design (CAD) models. An example of 3D-printing technology is fused deposition modeling (FDM), a fast and cost-effective filament-based method which builds objects layer by layer using extruded polymer melt. ${ }^{9}$ The most widely used material in FDM printers is polylactic acid (PLA), ${ }^{9}$ a well-known biocompatible thermoplastic polyester for vascular applications. ${ }^{10} \mathrm{FDM}$ is

Received: February 12, 2019

Accepted: March 25, 2019 


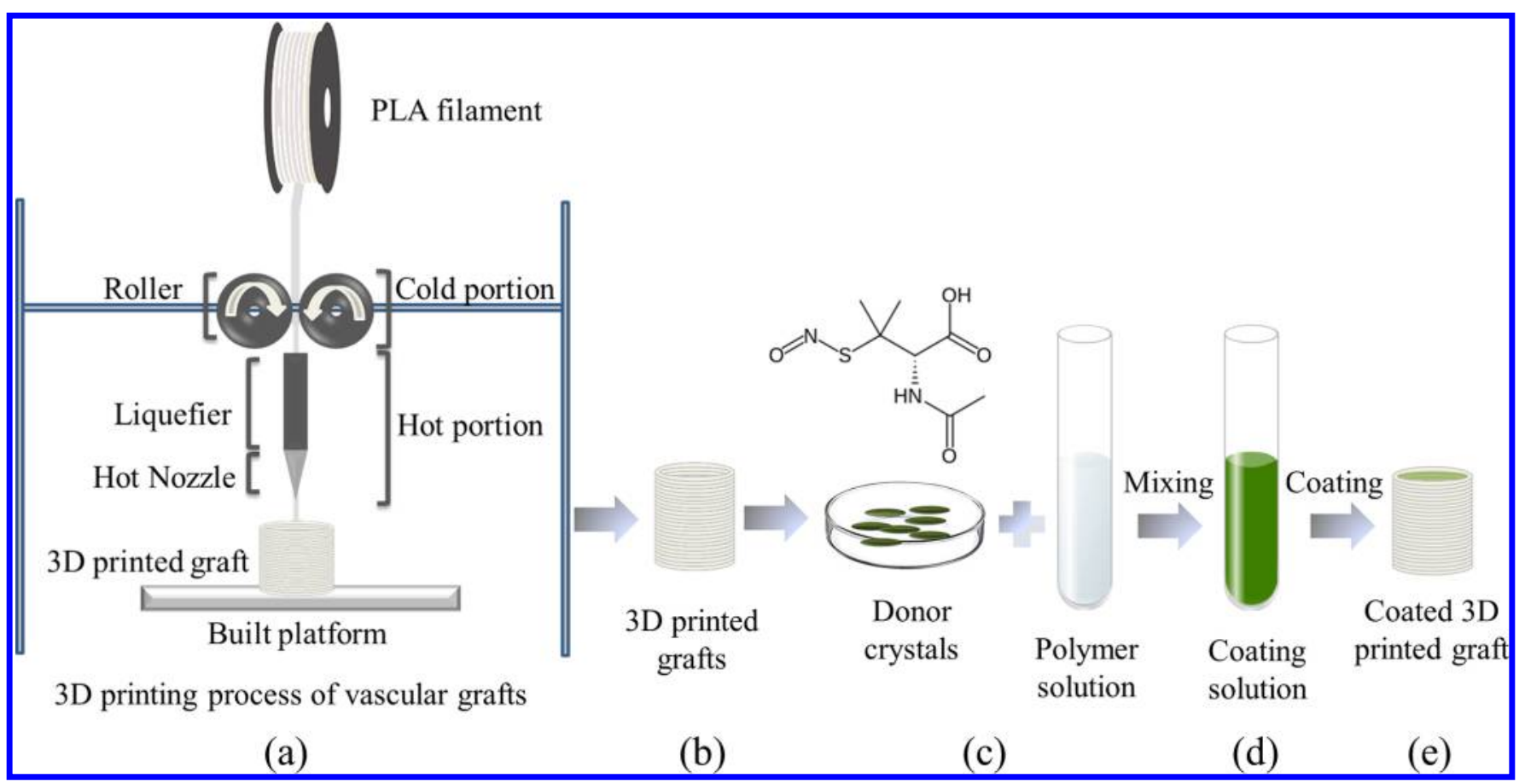

Figure 1. Schematic representation of sequential steps of manufacturing the NO-releasing 3D-printed vascular graft. (a) Printing of graft layer by layer using PLA filament and a FDM machine. (b) Prepared 3D-printed PLA graft. (c) Synthesized SNAP as NO donor with green crystals and chemical structure shown above were mixed with polymer solution. (d) Final coating solution of 10 wt \% SNAP in polymer (PEG, PCL, or PEGPCL). (e) Coated 3D-printed graft with coating solution ready for top-coating and biological experiments.

suitable for the printing of objects, using Food and Drug Administration (FDA) approved polymers such as PLA, without any need of chemical modification. This constitutes an advantage for 3D-printed objects for approval by the FDA. $^{11}$

Current synthetic commercial vascular grafts, especially grafts with an internal diameter of less than $6 \mathrm{~mm}$, manifest a high incidence of failure due to thrombus formation and bacterial infections. ${ }^{2,12-14}$ Therefore, there is a growing demand for engineered small-diameter vascular grafts (SDVG) with improved biocompatibility and lesser thrombogenicity. ${ }^{14}$ Staphylococcus aureus, Staphylococcus epidermidis, and Escherichia coli are the main pathogens responsible for medical device-associated infections. ${ }^{15}$ To generate an antibacterial surface, a common approach is the attachment of antibiotic molecules onto biomaterial surfaces to prevent bacterial growth. ${ }^{16,17}$ However, there is a need for a controlled antibiotic release system which can prevent bacterial infection and circumvent the secondary development of antibiotic resistance. ${ }^{17}$ Another approach in the construction of antimicrobial surfaces is based on the use of silver nanoparticles, which can be cytotoxic by inducing oxidative stress. ${ }^{18}$ Thrombosis represents another challenge of SDVGs. A common approach to prevent clot formation on blood contact surfaces is to dope them with antithrombotic agents. For example, heparin is a commercially available anticoagulant drug, which can prevent platelet adhesion and coagulation and consequently also limits the chance of infection by reducing bacterial entrapment within fibrin clots. ${ }^{19}$ In spite of these benefits, heparin cannot annihilate bacterial adhesion and may potentially cause serious adverse effects such as hemorrhage in surgical patients. $^{20}$

Nitric oxide (NO) is an endogenous bactericide and antithrombotic molecule, which might be a well-suited alternative agent to cover biomaterials in the development of antibacterial and hemocompatible surfaces, without side effects, because of its short half-life, precluding it from diffusion in blood and tissues. S-Nitrosothiols (RSNOs) such as $S$-nitroso- $N$-acetyl-D-penicillamine (SNAP) are biocompatible and endogenous types of NO donors and physiological transporters of NO in vivo. SNAP is derived from penicillamine, which is an FDA approved chelating agent and is one of the most stable NO-donors, due to intramolecular hydrogen bonding. $^{21-23}$ The mechanism of NO release from RSNOs, containing a nitrosonium group placed on sulfur and a thiol molecule, is mediated via the $\mathrm{S}-\mathrm{N}$ bond cleavage induced through heat or light radiation, which leads to formation of $\mathrm{NO}$ and thiyl $\left(\mathrm{RS}^{\bullet}\right)$. The generated $\mathrm{RS}^{\bullet}$ will react with another RSNO molecule to produce more NO and disulfide (RSSR). ${ }^{21}$

A widely used approach to control the NO-releasing rate is incorporation of polymers such as poly(ethylene glycol) (PEG) and polycaprolactone (PCL) with RSNOs as donorstoring agents. ${ }^{24,25}$ PEG is a well-known hydrophilic polymer, forming water-swollen networks, ${ }^{26,21}$ and polycaprolactone (PCL) is a hydrophobic, semicrystalline, and biocompatible linear aliphatic polyester, which is slowly degradable, and both are FDA approved. ${ }^{27,28}$ Blending of PEG and PCL in the solvent can reduce the interfacial tension of the solution. Therefore, the shrinkage of coating after solvent evaporation would be greater in a PEG-PCL layer compared to a pure PCL layer, and consequently the porosity of the PEG-PCL layer would be higher. ${ }^{29}$ The formed porous microstructure is advantageous for diffusion and entrapment of PBS to enhance water uptake and facilitate the NO release. Thus, the NOrelease mechanism via PCL erosion in a pure PCL layer will shift to PCL erosion and PBS diffusion. By exploiting the characteristics of these two polymers, we hypothesized that blending them could improve the biodegradability and water 
absorption and thus would further optimize the NO-controlled release kinetics.

Therefore, the aim of this study was to fabricate a novel 3Dprinted antibacterial and hemocompatible SDVG with controlled NO-release. We hypothesized that blending of hydrophilic and fast degrading PEG with hydrophobic and slow degrading PCL would make a suitable polymeric matrix to achieve sustained NO release. To test this hypothesis, 3Dprinted PLA vascular grafts were coated with PEG, PCL, or PEG-PCL (1:1), containing 10\% NO donor. In addition, we hypothesized that due to the hydrophobicity and slow degradation rate of PCL, a PCL top coat $(\mathrm{tc})$ could reduce the burst release of NO from the underlying coating. We then validated our hypothesis by studying the antimicrobial effect of the NO-releasing grafts during incubation with three different Gram-positive and Gram-negative bacteria. Moreover, we investigated the hemocompatibility of our NO-releasing grafts by testing their effect on blood platelet aggregation.

\section{MATERIALS AND METHODS}

2.1. Materials. Polycaprolactone (PCL, $\mathrm{Mn}=80000$ ), BioUltra grade PEG $(\mathrm{Mn}=4000), N$-acetyl-D-penicillamine (NAP), sodium nitrate, vanadium(III) chloride, sulfanilamide, $N$-(1-naphthyl) ethylene diamine dihydrochloride (NEDD), and tryptic soy broth (TSB) were purchased from Sigma-Aldrich (St. Louis, MO). Sulfuric acid, ortho-phosphoric acid, hydrochloric acid, methanol, tetrahydrofuran (THF), and sodium nitrite were obtained from Merck. Luria broth and calcium and magnesium free Dulbecco's phosphate buffered saline (PBS, pH 7.4) were purchased from Invitrogen and Gibco, respectively.

2.2. Design and Preparation of 3D-Printed Scaffolds. Scaffolds were designed to be $10 \mathrm{~mm}$ in height and $4.5 \mathrm{~mm}$ in internal diameter with $0.2 \mathrm{~mm}$ wall thickness using the Rhinoceros 3D CAD modeling package and were saved in a stereolithography (STL) format. The STL file, which describes the size and geometry of scaffolds was sliced into layers and converted into a g-code via CURA slicing software (Ultimaker BV, Geldermalsen, The Netherlands). The g-code was imported to the homemade FDM 3D-printer (optimized in our lab) to fabricate tubular grafts (Figure 1). The 3Dprinter extruded PLA filaments $(1.75 \mathrm{~mm}$ in diameter) through a 220 ${ }^{\circ} \mathrm{C}$ nozzle with a $200 \mu \mathrm{m}$ internal diameter to print objects layer by layer onto a heated bed. The platform temperature was set at $64{ }^{\circ} \mathrm{C}$, infill density was $100 \%$, and flow rate of the polymer was $206 \mathrm{~mm} / \mathrm{s}$.

2.3. NO Donor Synthesis. A modified protocol for SNAP synthesis was used in this study. ${ }^{30}$ In total, $1000 \mathrm{mg}$ of NAP was dissolved in $25 \mathrm{~mL}$ of methanol. Next, $15 \mathrm{~mL}$ of $1 \mathrm{M} \mathrm{HCl}, 500 \mu \mathrm{L}$ of $\mathrm{H}_{2} \mathrm{SO}_{4}$ (98\%), and $724.5 \mathrm{mg}$ of $\mathrm{NaNO}_{2}$ were added and mixed, respectively. After $15 \mathrm{~min}$ of stirring and $45 \mathrm{~min}$ of ice-cooling, methanol was evaporated under air flow at room temperature (RT) and light protected conditions. The dark green crystals were rinsed and filtered by ice-cooled $\mathrm{mQ}$ water and dried by lyophilization. The final crystals were kept in the dark at $-20{ }^{\circ} \mathrm{C}$.

2.4. Preparation of NO-Releasing Coating. The coating solutions were prepared by dissolving $100 \mathrm{mg}$ of PEG, PCL, or PEG-PCL (1:1) and $10 \mathrm{wt} \%$ SNAP in $1 \mathrm{~mL}$ of THF (hereafter named throughout the manuscript PEG-SNAP, PCL-SNAP, and PEG-PCL-SNAP). Based on recent study results which showed a greater NO flux from a SNAP-PEG composite layer with a molecular weight of PEG 4000, PEG 4000 was used in the current study. ${ }^{19}$ The control grafts were coated with the same coating solution without SNAP (hereafter named PEG, PCL, and PEG-PCL). In total, $200 \mu \mathrm{L}$ of coating solutions was injected inside each rotating graft in two steps and the grafts were kept in the dark at room temperature (RT) for solvent evaporation. Before and after coating, the grafts were weighed to test whether they were coated with equal amounts. The extra tc layer was prepared by dip coating of the dried coated grafts in PCL solution (100 mg of PCL dissolved in $1 \mathrm{~mL}$ of THF) (hereafter named PEG-SNAP-tc, PCL-SNAP-tc, and PEG-PCL-SNAP-tc for NO-releasing grafts, and PEG-tc, PCL-tc, and PEG-PCL-tc for control grafts). Then grafts were vacuum-dried and stored at $-20{ }^{\circ} \mathrm{C}$.

2.5. Graft Characterization. 2.5.1. Scanning Electron Microscopy (SEM) Imaging. Grafts were cut longitudinally by a sharp scalpel blade. Specimens were loaded on supporting stubs using carbon stickers and sputter coated with chromium. The samples were kept inside a vacuum chamber and were visualized using SEM (Zeiss Sigma, Germany) at an accelerating voltage of $2 \mathrm{kV}$ with the secondary electron (SE2) detector.

2.5.2. Water Uptake Study. NO-releasing grafts made out of different polymeric matrixes with or without tc were weighed $\left(W_{0}\right)$ after lyophilization and incubated in $10 \mathrm{~mL}$ of PBS at $37^{\circ} \mathrm{C}$ in the dark. At different time points, samples were wiped dry with tissue paper and immediately weighed $\left(W_{t}\right)$. The percentage of water uptake was calculated using the following eq $1:{ }^{19}$

$$
\text { water uptake }(\%)=\left(W_{t}-W_{0}\right) / W_{0} \times 100
$$

2.6. NO-Release Measurement (PBS-Plasma). Since the SNAP can decompose at elevated temperature, the grafts were immersed in $10 \mathrm{~mL}$ of PBS (pH 7.4) and incubated at $37^{\circ} \mathrm{C}$ in $15 \mathrm{~mL}$ falcon tubes covered with aluminum foil. This condition is similar to that in blood vessels, where $\mathrm{NO}$ can release due to thermal decomposition at $37^{\circ} \mathrm{C}$ in the presence of moisture. At specific time points $(0.5,1,1.5,3,5,7$, $24,48 \mathrm{~h}$ and up to 14 days), $150 \mu \mathrm{L}$ samples were collected for NOmeasurements. Since the half-life time of NO (a few seconds) limits direct measurement of $\mathrm{NO}$, its stable end products $\left(\mathrm{NO}_{x}\right.$, nitrate/ nitrite) were measured. The amount of $\mathrm{NO}_{x}$ released from the samples was evaluated using the modified Griess assay with a detection limit of $0.05 \mu \mathrm{M}^{13,31,32}$ Briefly, $50 \mu \mathrm{L}$ of sample and $50 \mu \mathrm{L}$ of $1: 1$ mixture of $2 \mathrm{~g}$ of sulfanilamide in $100 \mathrm{~mL}$ of $\mathrm{H}_{2} \mathrm{O} \mathrm{mQ}$ water with $3.44 \mathrm{~mL}$ of $\mathrm{H}_{3} \mathrm{PO}_{4}(85 \%)$ and $0.2 \mathrm{~g}$ of NEDD in $100 \mathrm{~mL}$ of $\mathrm{H}_{2} \mathrm{O}$ $\mathrm{mQ}$ water were loaded in each well of a 96-well flat bottom ELISA plate and gently mixed for $15 \mathrm{~min}$ in the dark. Next, $50 \mu \mathrm{L}$ of $\mathrm{VCl}_{3}$ solution, prepared by dissolving $400 \mathrm{mg}$ of $\mathrm{VCl}_{3}$ in $50 \mathrm{~mL}$ of $1 \mathrm{M}$ $\mathrm{HCl}$, was transferred to the well. The plate was covered with an adhesive film (VWR) and incubated at $60^{\circ} \mathrm{C}$ for $60 \mathrm{~min}$ in darkness. Afterward, the plate was cooled down and the absorbance $(550 \mathrm{~nm})$ was measured. The $\mathrm{NO}_{x}$ concentration was calculated using standard curves generated from serially diluted $\mathrm{NaNO}_{2}$ solutions. NO release in the presence of human plasma, pooled from six healthy donors was also measured as described above.

2.7. Degradation Study. Based on the results of the NO release, the PEG-PCL-SNAP-tc was selected for further stability studies. Therefore, the degradation kinetics of this graft were evaluated in vitro by weight loss monitoring of six samples. Briefly, the grafts were dried in an oven at $37{ }^{\circ} \mathrm{C}$ overnight, and the weight $\left(W_{0}\right)$ was measured. Afterward, the dried UV-sterilized grafts were immersed in sterile PBS, used as a degradation medium, and incubated at $37{ }^{\circ} \mathrm{C}$ in amber vials. At specific time points, the specimen were removed from the media, rinsed three times with distilled water to remove any degradation residues and dried at $37^{\circ} \mathrm{C}$ for $24 \mathrm{~h}$. After weighing $\left(W_{t}\right)$, the specimen were UV-sterilized and again soaked in degradation solutions. The degradation (\%) was calculated using the following eq $2:^{33}$

$$
\text { weight } \operatorname{loss}(\%)=\frac{\left(W_{0}-W_{t}\right)}{W_{0}} \times 100
$$

2.8. Graft-Bacterial Interaction Experiment. 2.8.1. Bacterial Culture Preparation. Bacterial strains stored at $-80{ }^{\circ} \mathrm{C}$ as glycerol stocks $(15 \%)$ were cultured on lysogeny broth (LB) agar (E. coli) or Mueller-Hinton blood agar plates (S. aureus and S. epidermidis). Prior to the experiment, single colonies of bacteria were picked up as an inoculum. S. aureus and S. epidermidis were grown in $10 \mathrm{~mL}$ of tryptic soy broth (TSB), and E. coli was grown in $10 \mathrm{~mL}$ of LB overnight $(\mathrm{ON})$ at $37^{\circ} \mathrm{C}$. The optical densities (OD) of the ON cultures were measured spectrophotometrically at $600 \mathrm{~nm}$ (UV-vis) after washing with sterile $\mathrm{PBS}$ and vortex mixing. The $\mathrm{OD}_{600}$ of bacterial solutions was measured and adjusted to the equivalent of $10^{7} \mathrm{CFU} \mathrm{mL} \mathrm{mL}^{-1.34}$ 


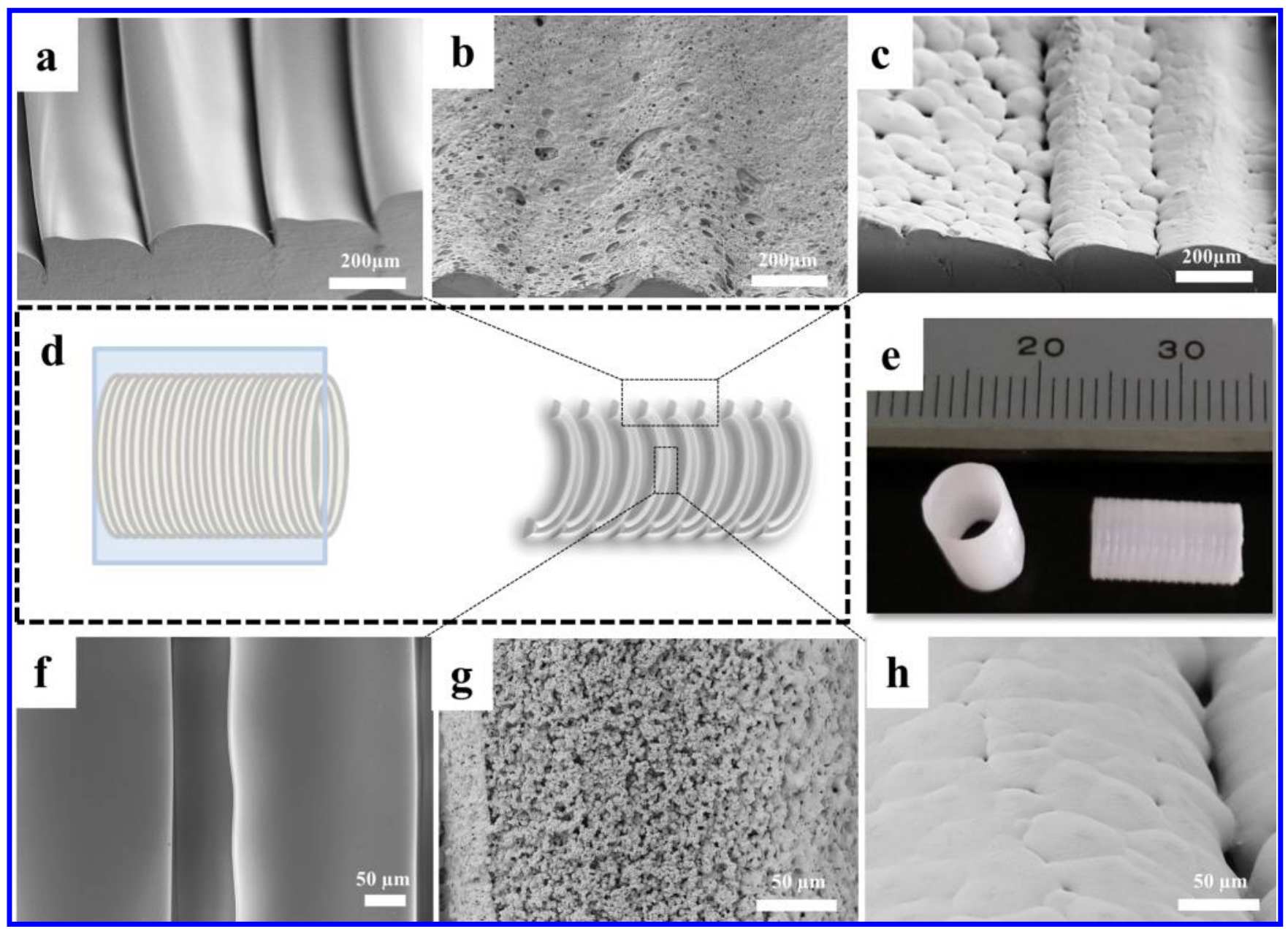

Figure 2. SEM images of the fabricated vascular grafts. SEM images from marginal portion of longitudinal sections and from the luminal surface of (a, f) bare PLA struts of 3D-printed vascular grafts, (b, g) coated 3D-printed PLA struts with PEG-PCL-SNAP, (c, h) same NO coated struts after addition of PCL topcoat: PEG-PCL-SNAP-tc. (d) Schematic longitudinal sectioning of vascular grafts and cut portion with marked area corresponding to the area of SEM images and (e) macroscopic top and side view images of 3D-printed PLA specimen. Scale bars: (a-c) $200 \mu \mathrm{m}$ and $(\mathrm{f}-\mathrm{h}) 50 \mu \mathrm{m}$.

2.8.2. Bactericidal Activity Analysis. The effect of NO release was investigated on the Gram-positive S. aureus ATCC 8325-4 and S. epidermidis ATCC 149900 and the Gram-negative E. coli ATCC 25922.

A single colony of each strain was cultured overnight in $10 \mathrm{~mL}$ of TSB (S. aureus and S. epidermidis) or LB (E. coli) at $37^{\circ} \mathrm{C}$. After bacterial washing with PBS and OD measurement, each strain was inoculated in PBS $+10 \%$ growth media with cell counts of $\sim 10^{7} \mathrm{CFU}$ $\mathrm{mL}^{-1}$ to detect the effect of NO-release and to rule out a lack of nutrition during the total length of the experiment, i.e., up to $72 \mathrm{~h}$. First, fresh NO-releasing grafts and control grafts without NO donor were tested and compared. Second, to eliminate the initial burst release, NO-releasing grafts and control grafts without NO donor were first presoaked in PBS for $24 \mathrm{~h}$ and then incubated in $2 \mathrm{~mL}$ of bacterial solution at $37{ }^{\circ} \mathrm{C}$ under shaking at $200 \mathrm{rpm}$, in the dark. At given time points of $3,6,24$, and $48 \mathrm{~h}, 100 \mu \mathrm{L}$ aliquots from each tube were collected and serially diluted in sterile PBS for colony counting on blood agar plates (S. aureus and S. epidermidis) and LB agar (E. coli). Experiments were repeated six times.

2.9. In Vitro Hemocompatibility Assay. The hemocompatibilities of PEG-PCL-SNAP-tc coated and PEG-PCL-tc control grafts were investigated by studying the activation and aggregation of human platelets. Fresh human platelet-rich plasma (PRP) was collected by centrifugation of human whole blood from healthy donors at $150 \mathrm{~g}$ for $10 \mathrm{~min}$ in sodium citrate tubes. Platelet poor plasma (PPP) was obtained by centrifugation of the remaining blood after removal of PRP at $800 \mathrm{~g}$ for $15 \mathrm{~min}$. Concentration of platelets in PRP was adjusted to $300 \times 10^{3}$ cells $/ \mu \mathrm{L}$ using PPP. To evaluate platelet aggregation, fresh NO-releasing grafts and 24 or $48 \mathrm{~h}$ presoaked grafts in PBS and the equivalent control grafts were incubated with PRP for different durations. ${ }^{35}$ Platelet aggregation was monitored by a fourchannel Chronolog optical aggregometer with Aggrolink software for $1 \mathrm{~min}$ as a baseline and continued upon addition of $10 \mu \mathrm{M}$ ADP to induce platelet aggregation under magnetic stirring at $37^{\circ} \mathrm{C}$. PPP was used as blank for aggregation tests and results were expressed as a percentage of maximal aggregation induced by ADP.

To study platelet activation morphologically, grafts were incubated in $1 \mathrm{~mL}$ of fresh PRP for $1 \mathrm{~h}$. Afterward, specimen were rinsed three times in PBS, and samples were fixed in $2.5 \%$ glutaraldehyde solution at $37{ }^{\circ} \mathrm{C}$ overnight. The samples were consequently dehydrated with gradient ethanol and dried at $37{ }^{\circ} \mathrm{C}$ overnight. The morphology of platelets was inspected by SEM after sputter coating with gold.

2.10. Statistical Analysis. All data were expressed as mean \pm standard deviation (SD). One-way or two-way analysis of variance (ANOVA) was performed for statistical analysis and followed by Tukey or Bonferroni post-test, respectively. The log-linear regression with $R^{2}$ and $P$ calculation was performed for the degradation analysis. $P<0.05$ was considered significant.

\section{RESULTS}

3.1. Fabrication and Characterization of 3D-Printed Grafts. 3D cylindrical vascular grafts were successfully printed using PLA filament and FDM, a fabrication technique, 


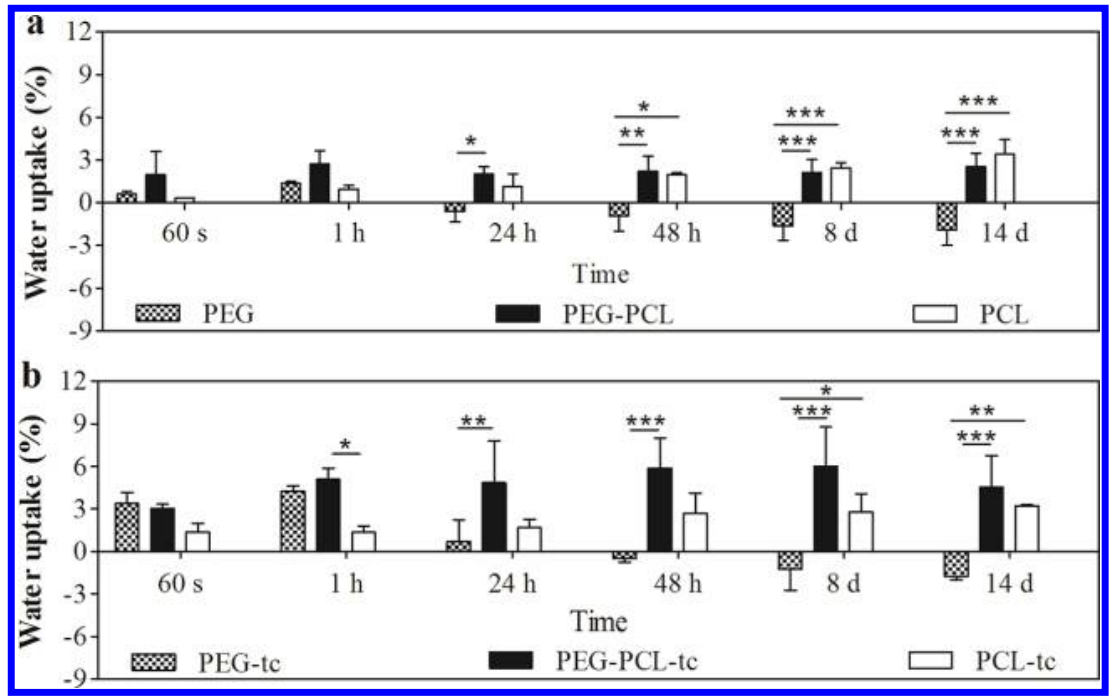

Figure 3. Water uptake (\%) of 3D-printed grafts. The specimens were kept in PBS for 14 days at $37^{\circ} \mathrm{C}$, and the percentage of water uptake was measured at different time points. Representative grafts were coated with PEG-SNAP, PEG-PCL-SNAP, and PCL-SNAP without tc (a) and with tc (b). Data are shown as mean $\pm \mathrm{SD}(n=6)$. $*=P<0.05$. ** $=P<0.01 . * * *=P<0.001$.

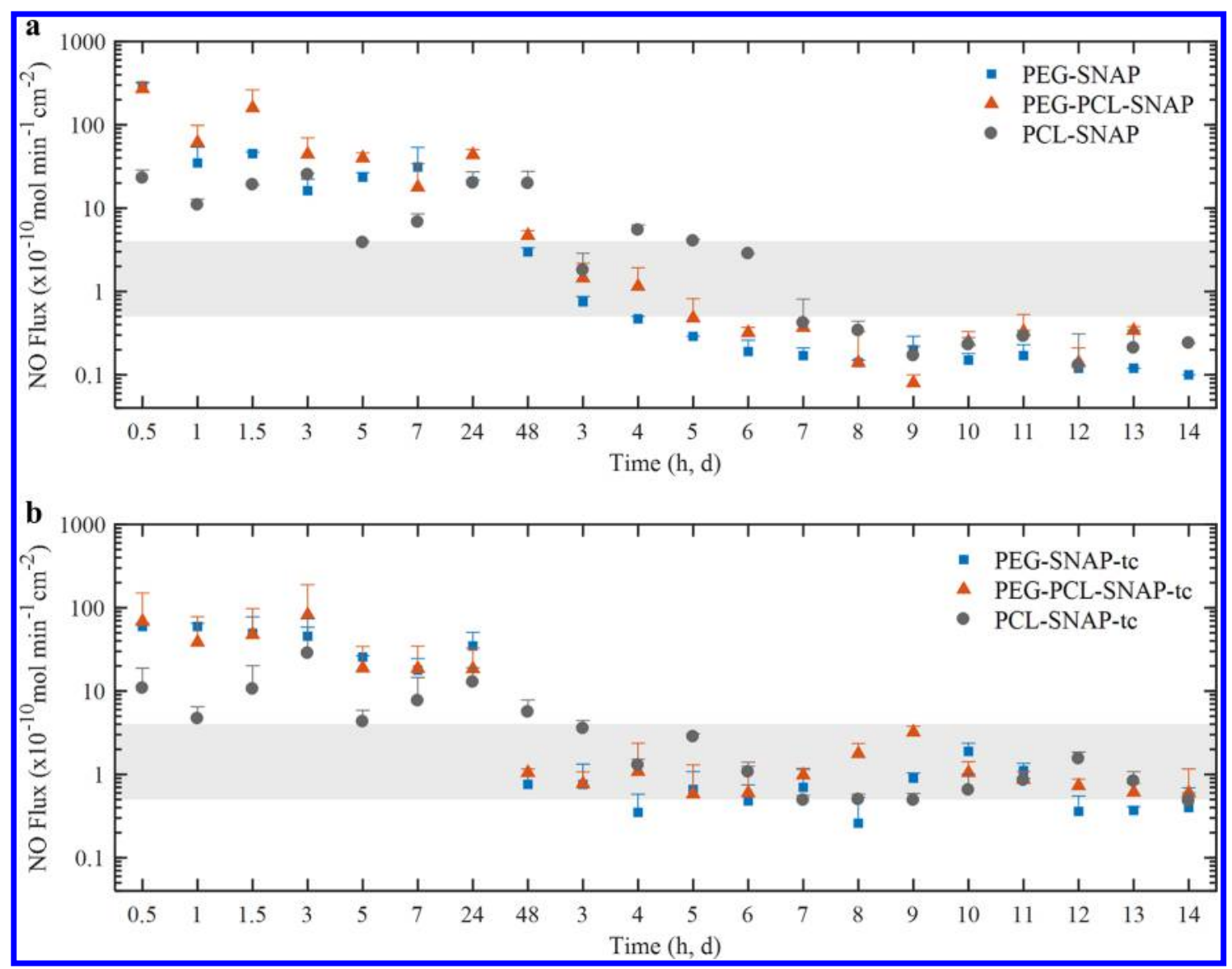

Figure 4. NO-release profile from coated 3D-printed grafts. NO-flux in PBS at $37^{\circ} \mathrm{C}$ from PEG-SNAP, PEG-PCL-SNAP, and PCL-SNAP from (a) grafts without tc, (b) and same grafts with tc over a 14 day period. The gray band represents the physiological range of $\mathrm{NO}$ flux $\left(0.5-4.0 \times 10^{-10}\right.$ $\left.\mathrm{mol} \mathrm{cm}{ }^{-2} \mathrm{~min}^{-1}\right)$. The $x$-axis represents the time which is in hours (h) and days from 0.5 to $48 \mathrm{~h}$ and 3-14 days, respectively. All NO amounts were measured by modified Griess assay. Data are expressed as mean $\pm \mathrm{SD}(n=6)$.

constructing objects layer by layer according to CAD data. The theoretical internal diameter (i.d.) was $4.5 \mathrm{~mm}$, and the actual i.d. was $4.47 \pm 0.064 \mathrm{~mm}$. The average weight of the grafts with a length of $10 \mathrm{~mm}$ was $52.18 \pm 0.78 \mathrm{mg}$, and the average wall thickness was $0.2 \pm 0.04 \mathrm{~mm}$.
3.1.1. Microscopic Observations. The luminal surface and the morphology of the 3D-printed grafts, coated and topcoated grafts were inspected by SEM (Figure 2d). SEM images reveal a circular and homogeneous morphology of the struts (Figure 2a,f). 


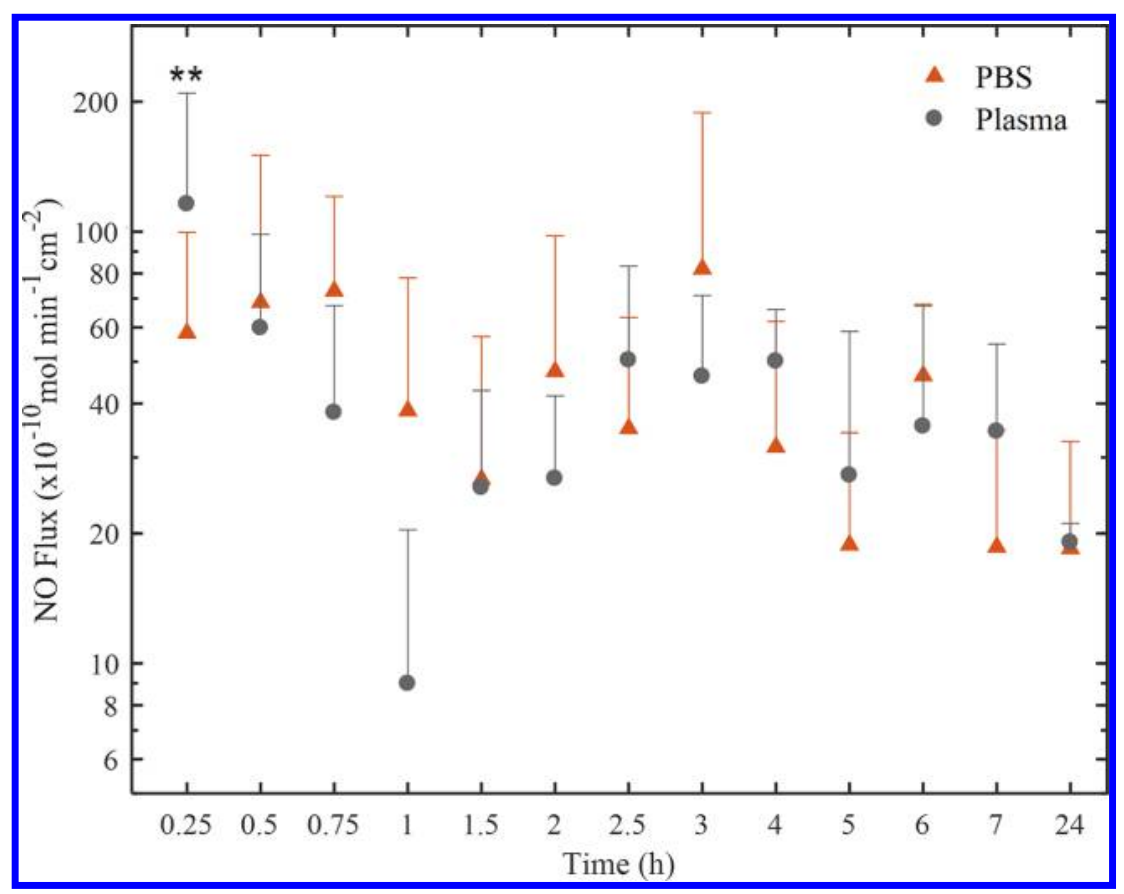

Figure 5. Comparison of NO-release profile in PBS versus plasma. NO flux from PEG-PCL-SNAP-tc in PBS versus plasma at $37{ }^{\circ} \mathrm{C}$. All NO amounts were measured by the modified Griess assay. Data are expressed as mean $\pm \mathrm{SD}(n=6)$. ** $=P<0.01$.

Figure 2b,g illustrates the PEG-PCL-SNAP composite coating covering the lumen of $3 \mathrm{D}$-printed grafts by which the surface morphology is changed from a smooth surface toward a rough morphology. A further change in surface morphology after additional tc is shown in Figure $2 \mathrm{c}, \mathrm{h}$. Comparing Figure 2b,g and Figure 2c,h, by incorporating PEG and NO-donor to PCL, the morphology of PCL was changed to a more irregular and porous structure. Figure $2 \mathrm{~b}, \mathrm{c}, \mathrm{g}, \mathrm{h}$, visualizes that the coating is homogeneously covering the PLA surface, which means that coating layers are evenly distributed on the struts and interstrut grooves.

3.1.2. Water Uptake. After coating and drying of each layer, 3D-printed grafts showed a similar weight, confirming uniform coating (Table S1).

Investigating the water absorption rate of coatings showed that the PEG-SNAP matrix absorbed water during the first hour of the experiment (Figure 3a). However, after $24 \mathrm{~h}$ it loses weight due to polymer washout. On the other hand, the PCL-SNAP matrix demonstrated continuous water uptake until the end of the experiment on day 14. Blending of PEG, PCL, and SNAP prevented excessive degradation of PEG and, therefore, resulted in the highest water uptake until day 2, followed by degradation of the PEG in the polymer matrix.

Adding the tc layer over PEG-SNAP prevented the degradation of PEG during the first hours of the experiment (Figure 3b). However, after $48 \mathrm{~h}$, washout was seen, which triggered weight loss. The water uptake of PCL-SNAP-tc was similar to that for PCL-SNAP. Considering all experimental conditions, the maximum water uptake was obtained from PEG-PCL-SNAP-tc (Figure 3).

3.2. NO-Release. In a pilot experiment, we prepared the same coatings with $5,10,15$, and $20 \mathrm{wt} \% \mathrm{NO}$-donor to test the amount of NO release. The results of $10 \%$ donor coating exhibited NO release in the physiological range of $0.5-4.0 \times$ $10^{-10} \mathrm{~mol} \mathrm{~cm}^{-2} \mathrm{~min}^{-120,36}$ (Figure S1). Although, the initial burst release of $5 \mathrm{wt} \% \mathrm{SNAP}$ produced NO-concentrations closer to the physiological range, the SNAP reservoir was not adequate enough to support optimal release for 2 weeks, as the flux dropped to the border of the physiological range at $72 \mathrm{~h}$. Therefore, the coating with $10 \%$ NO donor was used for all experiments of the current study.

The results of NO-release from 3D-printed grafts with different types of polymer matrixes, such as PEG-SNAP, PEGPCL-SNAP, and PCL-SNAP, were investigated with and without tc. PEG showed an extreme NO release in the early minutes of the experiment, and the NO flux reached the physiological range only on days 2 and 3. Similar results with a somewhat longer and smaller burst release were obtained for the PEG-PCL-SNAP polymer matrix where the NO release was in the physiological range on days 3-5. However, the NO burst release from the PCL-SNAP matrix on day 1 was much lower than in PEG-SNAP and PEG-PCL-SNAP, and the NO flux reached the physiological range at $5 \mathrm{~h}$, days 3,5 , and 6 (Figure 4a).

In general, by adding the tc layer to all matrixes (Figure 4b), the NO burst release was reduced and the duration of NO release in the physiological range increased remarkably from 24 to $48 \mathrm{~h}$ onward. However, the NO burst release was higher in PEG-SNAP-tc and PEG-PCL-SNAP-tc matrixes compared to PCL-SNAP-tc during the first $24 \mathrm{~h}$ but lasted longer in the PCL-tc matrix $(48 \mathrm{~h})$. Nonetheless, the NO-flux from the three coatings with tc were not significantly different after $24 \mathrm{~h}$, PEG-PCL-SNAP-tc was the only matrix showing a physiological level of NO flux until day 14.

NO-release from the PEG-SNAP-tc matrix was in the physiological range between $48 \mathrm{~h}$ and day 3 , nevertheless fluctuating between values in the physiological range or even lower afterward. The NO flux from PEG-PCL-SNAP-tc was in the physiological range from $48 \mathrm{~h}$ to day 14 and the one from PCL-SNAP-tc between day 3 and 14, respectively. Results of NO-release are shown in detail in Figure 4.

Since the results of the NO release from PEG-PCL-SNAP-tc showed the relatively longest $\mathrm{NO}$ release in the physiologic range, this blended graft was further evaluated for its NO- 


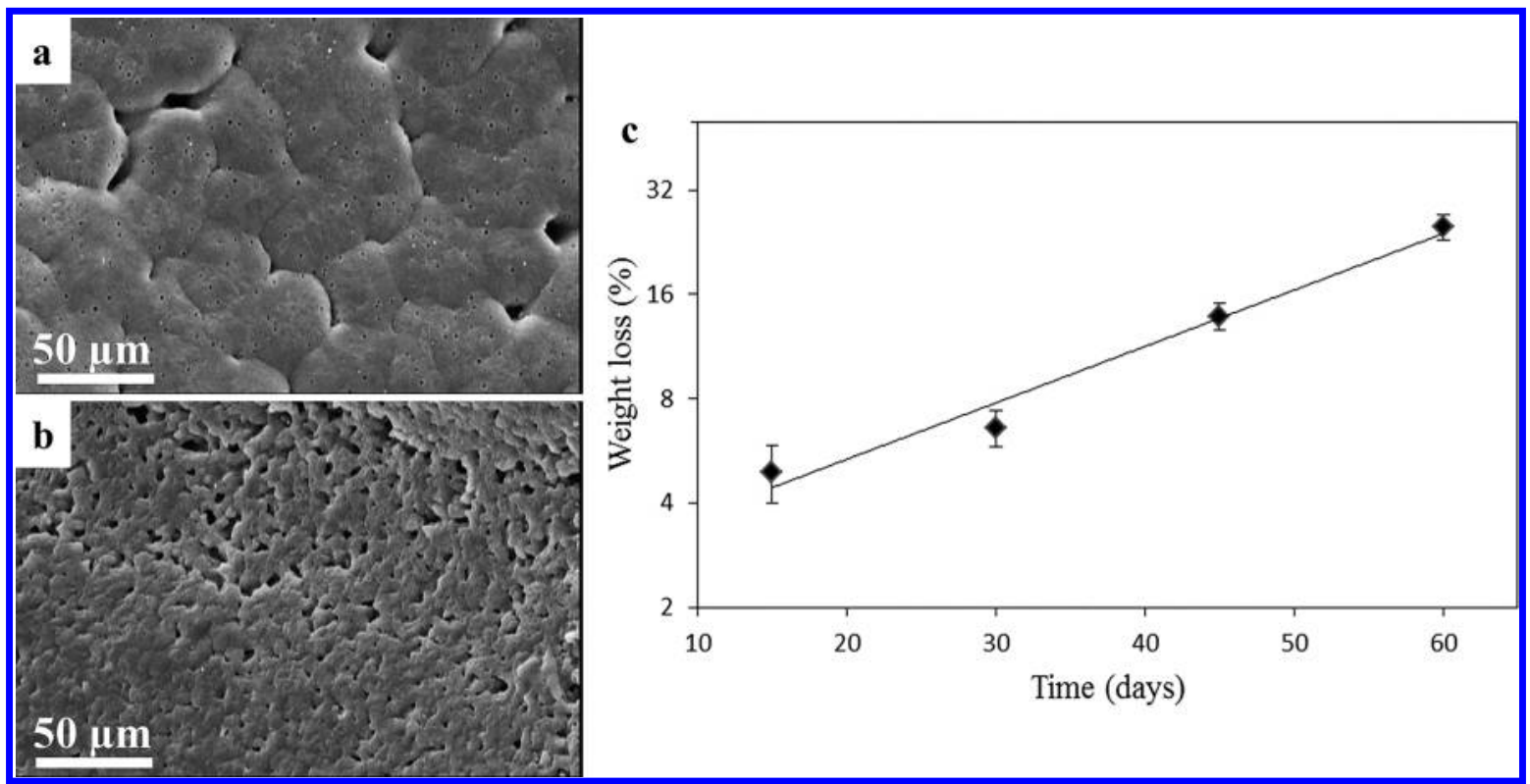

Figure 6. Degradation rate of grafts coated with PEG-PCL-SNAP-tc over 60 days. Representative SEM images of degradation after (a) 15 days and (b) 60 days incubation in PBS at $37^{\circ} \mathrm{C}$. (c) Percentage of weight loss was measured in PBS at $37{ }^{\circ} \mathrm{C}$, which revealed a linear degradation in a logarithmic scale. Data are expressed as mean \pm SD $(n=6) . R^{2}=0.975$.

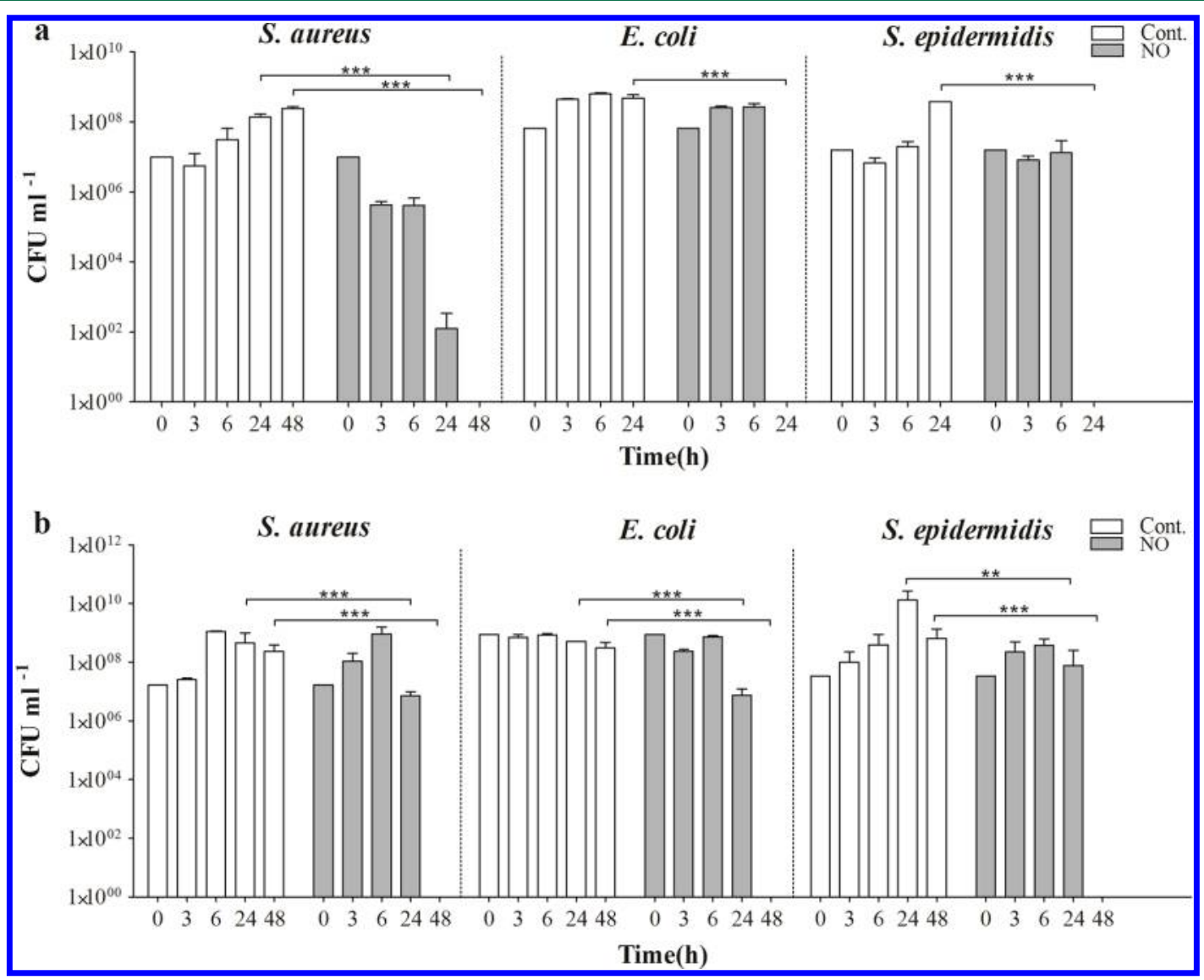

Figure 7. Effect of NO release from 3D-printed grafts on bacterial viability. Plate counting of the number (CFU $\left.\mathrm{mL}^{-1}\right)$ of viable $S$. aureus, E. coli, and S. epidermidis incubated with (a) fresh NO-releasing and control grafts and (b) with $24 \mathrm{~h}$ presoaked NO-releasing and control grafts. The data were collected at the beginning of experiment $(0 \mathrm{~h})$ and at $3,6,24$, and $48 \mathrm{~h}$ of incubation. Data are expressed as mean \pm SD $(n=6)$. Cont. $=$ Control. $* *=P<0.01, * * *=P<0.001$.

releasing properties in human plasma, a setting closer to the in vivo situation. Results illustrate that plasma does not remarkably diminish NO release from blended grafts. Despite a higher early burst NO release in plasma at $15 \mathrm{~min}$, overall, the NO release in plasma and PBS are not significantly different (Figure 5). 


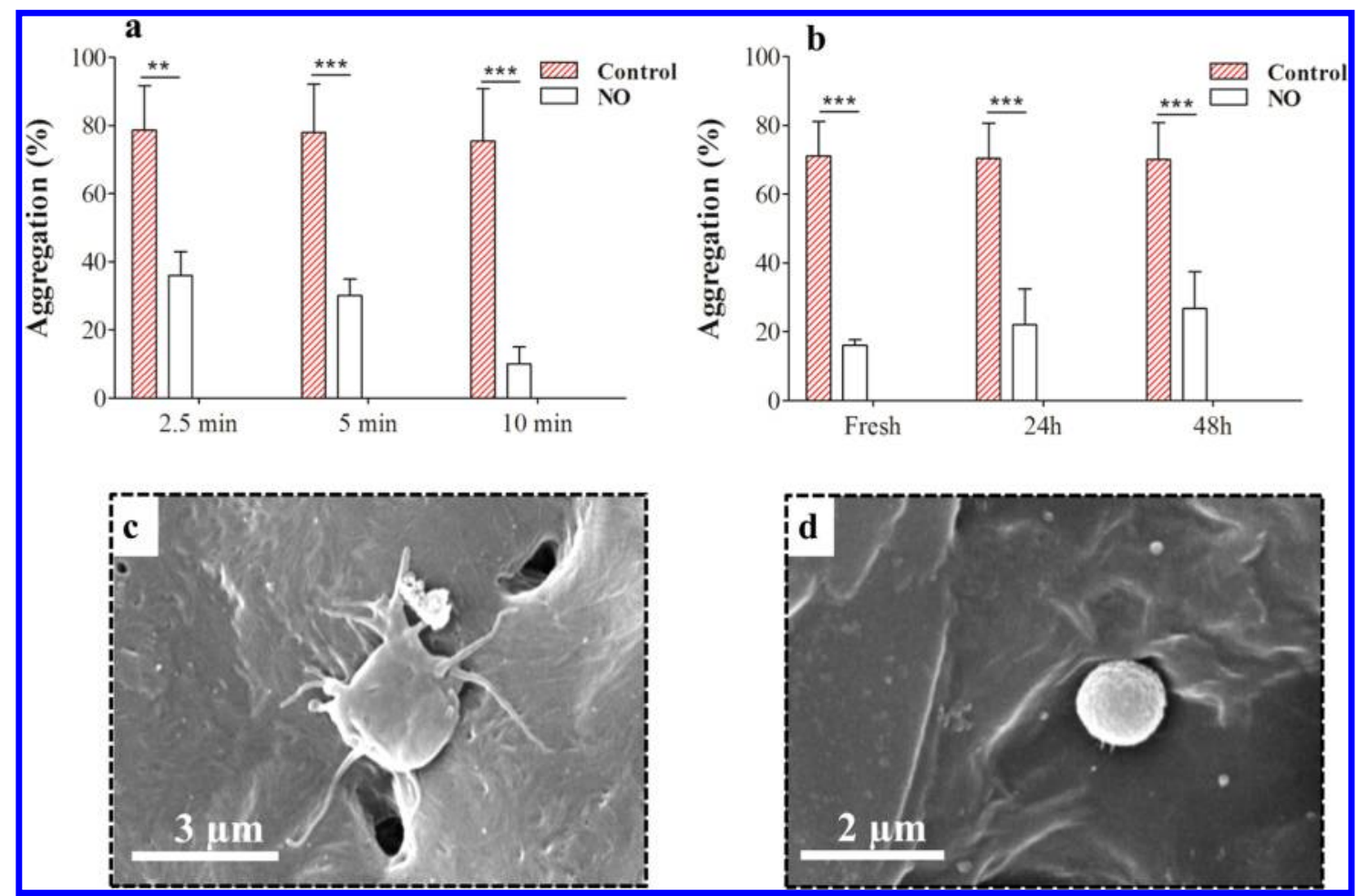

Figure 8. Antiplatelet aggregation and activation effects of NO releasing grafts. Aggregation (\%) of fresh human PRP of three donors incubated with (a) fresh grafts for 2.5, 5, and $10 \mathrm{~min}$ and (b) fresh, $24 \mathrm{~h}$, and $48 \mathrm{~h}$ presoaked grafts for $10 \mathrm{~min}$. Morphology of graft-adhered platelets after $1 \mathrm{~h}$ incubation of fresh human PRP with (c) PEG-PCL-tc coated control grafts and (d) PEG-PCL-SNAP-tc coated grafts, which demonstrated the activated irregular morphology with protruded pseudopodia and resting spherical morphology, respectively. The statistical data were obtained by comparing the value of each condition with the proper control value. Data are expressed as mean $\pm \operatorname{SD}(n=3)$. ** $=P<0.01$. *** $=P<0.001$.

3.3. In Vitro Degradation Study. It has been reported that the healing process of vascular grafts, until a complete EC monolayer covers the artificial surface, takes approximately 6 weeks. ${ }^{37}$ Therefore, in order to evaluate whether the stability of the grafts with a PEG-PCL-SNAP-tc coating would support vascular regeneration during 6 weeks, their degradation rate was studied. The degradation of polyesters results from hydrolysis and diffusion of liquid molecules into the samples and causes degradation of the polymer chain over time. ${ }^{38,39}$ The microscopic inspection of grafts after 15 days of incubation displayed the beginning of degradation by formation of very small holes on the surface (Figure 6a), which progressed to larger holes after 60 days of degradation (Figure 6b)

At intervals of 15 days, the weights of dried specimens were recorded. Figure $6 \mathrm{c}$ shows the weight loss kinetics of the grafts incubated in PBS at $37^{\circ} \mathrm{C}(n=6$ for each specimen) over a 60 day period. The degradation process corresponds to a monophasic decay, with the grafts undergoing a $25 \pm 2.08 \%$ mass loss over this 8.5 -week time interval and an estimated loss of $13 \%$ after 6 weeks.

3.4. Biological Effects of NO Release. 3.4.1. Bacterial Viability. 3.4.1.1. NO Released from Grafts Effectively Inhibits Bacterial Growth. To investigate the effect of NO release in PEG-PCL-SNAP-tc grafts and controls, both were incubated with $10^{7} \mathrm{CFU} \mathrm{mL} \mathrm{m}^{-1} \mathrm{~S}$. aureus, S. epidermidis, and E. coli under shaking conditions. CFU counting at different time points after NO-release revealed that $S$. epidermidis and E. coli were completely neutralized after $24 \mathrm{~h}$, whereas $99.99 \%$ of $S$. aureus were reduced at this time and no further growth was observed after $48 \mathrm{~h}$. In contrast, bacteria incubated with control grafts were proliferating to the expected steady-state level (Figure 7a).

3.4.1.2. Presoaked NO-Releasing Grafts Inherit Strong Bactericidal Properties. To avoid the effect of the initial high concentration of NO in PEG-PCL-SNAP-tc grafts, control and NO-releasing grafts were presoaked in PBS for $24 \mathrm{~h}$. Then, the soaking solution containing the initial high concentration of $\mathrm{NO}$ was discarded in order to switch to an NO flux in the physiological range and constant until day14. The bactericidal effect of NO from the presoaked grafts was investigated as it specifically reflects biological effects during longer-term exposure. The results revealed that the populations of all three strains were reduced significantly after $24 \mathrm{~h}(P<0.001$ for $S$. aureus and E. coli, $P<0.01$ for $S$. epidermidis), with no bacteria surviving after $48 \mathrm{~h}$ of incubation. Similar to the bacterial experiment with fresh grafts, for the control presoaked grafts, bacteria were likewise proliferating, reaching steady state numbers (Figure $7 \mathrm{~b}$ ). Overall even when presoaked blended grafts display milder features, these are still effective, executing rapid antimicrobial activity.

3.4.2. Platelet Aggregation Assay. 3.4.2.1. Effect of Incubation Time. In order to find the minimum duration of platelet incubation with $\mathrm{NO}$ which would reach maximal inhibition of aggregation, four incubation time points from 2.5 to $20 \mathrm{~min}$ were chosen. The blended grafts were incubated with fresh PRP from three different donors for 2.5, 5, 10, and $20 \mathrm{~min}$, and the ADP-induced platelet aggregation was investigated immediately. NO significantly reduced platelet aggregation after incubation with PRP for $2.5,5$, and $10 \mathrm{~min}$ in a progressive manner, showing the lowest values at $10 \mathrm{~min}$ (Figure 8a). Platelet aggregation at $20 \mathrm{~min}$ showed similar 
values $(10 \pm 1.8 \%)$ as for $10 \mathrm{~min}(10.25 \pm 0.35 \%)$, i.e., of about $10 \%$ residual aggregation. Therefore, the $10 \mathrm{~min}$ incubation time was selected as the optimal duration time to achieve maximal inhibition of platelet aggregation.

3.4.2.2. Presoaking of NO-Grafts Does Not Interfere with Its Very Effective Antiplatelet Activity. Based on the results of different incubation times (Figure 8a), the 10 min incubation was selected to investigate if presoaking grafts in PBS would likewise maintain their capability to inhibit platelet aggregation. Fresh, $24 \mathrm{~h}$, and $48 \mathrm{~h}$ presoaked grafts were incubated with fresh PRP for $10 \mathrm{~min}$. The percentages of platelet aggregation in all conditions were significantly lower than the respective control conditions, and residual platelet aggregation in the presence of presoaked grafts was not significantly different compared to incubations with fresh grafts (Figure $8 \mathrm{~b})$. Results for $48 \mathrm{~h}$ presoaked grafts showed also a significant inhibition of platelet aggregation for all time points (Figure S2). Overall, despite graft pretreatment, platelet aggregation is weakened to the same extent reflecting sufficiently high exposure to $\mathrm{NO}$ in all cases. In addition, reaction kinetics are immediate, with maximum inhibition already attained after incubation times of $10 \mathrm{~min}$.

3.4.3. Platelet Surface-Activation Study. The morphology of platelets after $1 \mathrm{~h}$ incubation with grafts was investigated by SEM imaging. While platelets adhering to control grafts were activated, i.e., showed an irregular shape with pseudopod formation (Figure 8d), platelets sticking on the NO-releasing grafts showed a discoid and nonactivated morphology.

\section{DISCUSSION}

Fabrication of vascular grafts using 3D-printing technology has been achieved with good results. ${ }^{40-45}$ However, failure of these small size grafts is common, due to infection and thrombotic complications after implantation. ${ }^{12-14}$ Therefore, we combined the 3D-printing technology with the properties of NO releasing systems to produce novel 3D-printed SDVGs with antibacterial and antithrombotic properties. By coating the 3Dprinted grafts, we reached a controlled release of $\mathrm{NO}$ in the physiological concentration range, capable of substantially diminishing bacterial growth and of inhibiting platelet functionality in their surroundings.

The 3D-printing method has the advantage of being costeffective, and it allows fast processing during the construction of custom-based geometries with high accuracy. In previous research, we have fabricated vascular structures from PLA with $200 \mu \mathrm{m}$ wall thickness using a FDM machine. ${ }^{33}$ For the first time, these PLA grafts were coated in the present study with a blend of PEG-PCL-SNAP NO releasing layer after fabrication, since $\mathrm{NO}$ donors are not stable during the extrusion through the $220{ }^{\circ} \mathrm{C}$ nozzle printer.

Our data show that the PEG-PCL-SNAP blend exhibits the most favorable water uptake and an extended NO release profile in the physiological range, an improvement compared to each matrix composite alone. This verifies that a better control of NO release could be reached by combining different polymer matrixes with different water uptake and hydrolysis profiles before coating onto the lumen of the 3D-printed grafts. NO release from SNAP by breakage of the S-NO bond was catalyzed by heat and humidity of the graft's incubation. It has been reported that by doping SNAP into polymers, SNAP molecules form crystals that manifest strong intermolecular interactions, resulting in a slow dissolution and release of NO. $^{46,47}$
Previous studies demonstrated the effect of hydrophobic polymers such as polyurethane $e^{16,20,46,27}$ or hydrophilic polymers such as $\mathrm{PEG}^{19}$ as a polymeric matrix for NO release. It is known that blending of PEG and PCL cannot only extend the degradation time of PEG but also can reduce the hydrophobicity of PCL. ${ }^{48}$ Recently, Chen et al. used PEG $(\mathrm{Mw}=20000)$ and PCL $(\mathrm{Mw}=80000)$ blends with various molar ratios $(\mathrm{PEG} / \mathrm{PCL}=1-9$ and $4-1)$ to construct drug eluting stents using electrospinning and electrospraying techniques. They explained that the hydrophilic PEG in PEG-PCL blends makes the aqueous phase and work as a pore former, which corresponds to formation of swollen portions with controlled drug releasing kinetics. ${ }^{49}$ This gave us the idea to create the new coating with a PEG-PCL matrix for controlled NO release. We hypothesized that PEG, as a very hydrophilic polymer, can absorb water into the polymeric matrix facilitating the dissolution of the NO donor. The results of the current study demonstrate that blending of a 1:1 ratio of PEG and PCL can control the PEG degradation rate and consequently regulate NO releasing kinetics.

The blend of the PEG-PCL-SNAP inspected by SEM imaging revealed a thin layer of coating giving a rough morphology on the lumen of 3D-printed PLA, which explains the kinetics of water uptake and NO-release. By making a PCL tc layer, this rough morphology is transformed into a smoother surface with some globular appearance. The tc layer also covers the gaps which influences the kinetics of NO-release and water uptake (Figure 2). Furthermore, it should be mentioned that THF was used to prepare coatings and the top-coat, since it is a noncytotoxic common solvent for dissolving PLA, PCL, PEG, and SNAP. It can be expected that during the coating process, THF can diffuse into and dissolve a thin superficial layer of the lower layer and therefore the layers will be stably fused into one another. ${ }^{50}$

Furthermore, the results indicate that PEG and PCL, as very hydrophilic and hydrophobic polymers, respectively, show different NO-release. In detail, released NO-levels from the PEG-SNAP matrix are maximal in the early minutes, whereas NO-release from PCL-SNAP is higher from $48 \mathrm{~h}$ onward. These results are mainly in agreement with the water uptake results of PEG-SNAP and PCL-SNAP matrixes (Figure 3). PCL-SNAP absorbs water gradually until day 14 followed by diffusion of NO, while PEG-SNAP absorbs water immediately and simultaneously begins to washout.

Interestingly, our results indicate that the water uptake and consequently the burst release of $\mathrm{NO}$ can be optimized and controlled by constructing a novel composite mixture of PEGPCL-SNAP (Figure 3 and Figure 4). In this 1:1 blended matrix, the PEG portion absorbs fluid which facilitates donor dissolution and NO-release while the PCL portion prevents rapid water uptake and washout of PEG. PCL-SNAP-tc reduced the NO release significantly and prolonged the duration of NO release. More importantly, our results demonstrate that our aim to dampen the initial burst release and to prolong NO release by the tc layer using the dip coating method was successful. This finding is in agreement with earlier studies reporting that covering the SNAP-reservoir coating with a hydrophobic tc layer can optimize the NO release kinetics. ${ }^{36,51}$ In Figure $4 \mathrm{a}$, the NO-releasing matrixes were not protected with the tc layer, hence causing a higher NO-release than in the matrixes with tc (Figure 4b). In Figure 4 , the gray band indicates the physiological range of $\mathrm{NO}$ 
release, which was aimed for in this study, being reached in the tc conditions only.

The bioavailability of $\mathrm{NO}$ under in vivo conditions is regulated by hemoglobin, which makes the half-life of $\mathrm{NO}$ as short as seconds. ${ }^{52}$ However, owing to its rapid disintegration, the initial burst release of $\mathrm{NO}$ in the first hours is not of major concern for medical implants in vivo. ${ }^{46}$

Investigating the NO release in an experimental situation closer to the in vivo situation uncovered that the amount of $\mathrm{NO}$ release is not significantly influenced in the presence of plasma (Figure 5). Once the biomaterials have contact with blood circulation, the surface is covered with plasma proteins. This protein coating known as "hard corona" firmly adsorbs on the surface and remains stably adhered. Due to the stability of the hard corona for the rest of the release period, the NOrelease in plasma was measured repetitively during the first 24 h. Behzadi at al. stated that the protein rich environment of plasma could cover and shield the biomaterial's surface and thereby reduce drug release. ${ }^{53}$ They promote a model of protein corona layer formation around nanoparticles, responsible for a reduction of drug release by shielding the surface. However, our current results do not provide evidence for the suggested model in our experimental setting which might be due to the use of a bulk surface instead of nanoparticles. Further analytical investigations are needed to clarify this model for NO release in plasma, especially when protective coats (tc) are present.

$\mathrm{NO}$ inherits antimicrobial properties due to denaturation of enzymes, deamination of DNA, and lipid oxygenation in a bacterial matrix. ${ }^{54}$ In that sense, NO would have a desirable preventive effect on graft infections after implantation. Therefore, the effect of NO on three different strains was examined by incubating the grafts with E. coli as a Gramnegative and $S$. aureus and $S$. epidermidis as the two most common Gram-positive strains in vascular grafts infection. Our results verify that the amount of NO released from the grafts is capable to eradicate all E. coli and S. epidermidis before $24 \mathrm{~h}$ and $99.99 \%$ of $S$. aureus before $24 \mathrm{~h}$. Together, this means that the bacterial load was reduced by 4 orders of magnitude and $S$. aureus even by $100 \%$ before $48 \mathrm{~h}$, while bacteria were proliferating without any growth limitation in the presence of control grafts. This initial protection, as previously reached by drug diluting stents, ${ }^{49}$ is therefore likely a concept relevant for vascular grafts.

Since the NO-releasing grafts and controls did not differ in their polymeric structure, the antibactericidal effect of NO is confirmed. These results go along with findings of other models where NO delivery successfully killed bacteria such as S. aureus, ${ }^{55-57}$ E. coli, ${ }^{55}$ and Pseudomonas aeruginosa. ${ }^{54-57}$

It seems that $S$. aureus has a somewhat higher (but not significantly different) resistance to $\mathrm{NO}$ in comparison to the two other strains, probably linked to the bacterial genome sequence of $S$. aureus. This has also been stated by Seabra et al. $^{57}$ who tested the viability of $S$. aureus and $P$. aeruginosa after incubation with their nitrosated synthesized films. Their results showed that $S$. aureus is more resistant to NO as $2 \%$ of bacteria survived after $24 \mathrm{~h}$.

The structural differences of the Gram-positive and Gramnegative strains cause different sensitivities to external substances such as antibiotics. ${ }^{58,59}$ Considering this difference in the bacterial cell envelope, results display that current 3Dprinted vascular grafts with controlled releasing of $\mathrm{NO}$ can kill both Gram-positive $S$. aureus and S. epidermidis and Gram- negative E. coli bacteria. To distinguish the effect of NO release from the effect of poor nutrition on bacterial growth, $10 \%$ growth media was added to PBS to allow bacterial growth. The initial concentration of bacteria was also selected between $10^{7}$ to $10^{8} \mathrm{CFU} \mathrm{mL} \mathrm{mL}^{-1}$, which is considerably higher than in other studies which used an initial concentration of $10^{5} \mathrm{CFU}$ $\mathrm{mL}^{-1} 55$

Seabra et al. argued that the initial burst release of NO may be beneficial to prevent the early stages of bacterial colonization and that prolonged NO-release at a lower level is needed to avoid bacterial growth. ${ }^{57}$ In the present study, we choose to also eliminate the effect of the NO-burst release by presoaking the grafts in PBS before bacterial incubation. Interestingly, results illustrate that the reached reduction in NO-release to a physiological range can still exponentially diminish bacterial survival up to $24 \mathrm{~h}$ and eradicate all bacteria before $48 \mathrm{~h}$ of incubation. Aiming to prevent graft infection, total eradication of bacteria seems important since residual bacterial growth could induce an infective state. Another advantage of NO is that it is able to diffuse in biofilms and by doing so can act on bacteria not directly bound to the graft. ${ }^{54}$

In addition, our study shows another important property of the novel construct of PEG-PCL-SNAP-tc in terms of prevention of graft thrombosis, especially an issue in SDVGs. Assessing platelet aggregation upon NO release by LTA, NOreleasing grafts displayed a significant reduction of $\mathrm{ADP}$ induced platelet aggregation in comparison to control grafts. These results correlate well with other studies showing inhibition of platelet aggregation in the presence of NO donors such as diazeniumdiolated dibutylhexanediamine $\left(\mathrm{DBHD}-\mathrm{N}_{2} \mathrm{O}_{2}\right){ }^{35,60}$ SNAP, ${ }^{61}$ and $S$-nitroso-glutathione doped with various polymeric matrixes. ${ }^{62}$

The effect of NO release on platelet aggregation was investigated in a time-dependent manner reaching a maximum effect after $10 \mathrm{~min}$ long incubations. A significant reduction of platelet aggregation was still found when the grafts were presoaked in PBS for 24 or $48 \mathrm{~h}$. These results demonstrate, even when the burst NO-release is eliminated via presoaking of grafts in PBS, that the degree of platelet aggregation is still considerably reduced. Hence, our NO-release data from PEGPCL-SNAP-tc, showing an NO release in the physiological range from $24 \mathrm{~h}$ up to day 14, indicate that PEG-PCL-SNAP-tc grafts execute antiplatelet aggregation effects, at least lasting 14 days.

Moreover, the effect of NO-release on the morphology of platelets was also investigated. The results (Figure $8 \mathrm{c}, \mathrm{d}$ ) revealed platelet activation in the control group, whereas in the NO-releasing condition, the platelets showed a discoid (resting) morphology. A plausible explanation for these results is the stimulation of platelet soluble guanylate cyclase (sGC) by released NO, resulting in cGMP-mediated inhibition of platelet activation, in agreement with previous studies. ${ }^{63}$

Overall, thrombosis and bacterial infection are undesired complications of vascular grafts in vivo. The first $6 \mathrm{~h}$ of postimplantation, also known as the "decisive period", are critical for the long-term effectiveness of an implanted graft. $^{64,65}$ During this period, the risk of thrombosis ${ }^{22}$ and bacterial infection could compromise tissue integration which determines the success or failure of implantation. ${ }^{65}$ Therefore, the potent early NO-release could promisingly prevent the failure of implanted SDVGs in the initial hours. Moreover, the later (physiological range) NO-release can promote the endothelialization process ${ }^{66}$ of endothelial cells (ECs), 
migrating from the cut-edge border of adjacent blood vessels to the SDEVG's lumen. ${ }^{66,6}$ This healing process, which takes approximately 6 weeks to create a complete ECs monolayer, ${ }^{37}$ may be facilitated by NO-release over an interval of 14 days, thus also offering a beneficial, longer-term clinical prognosis. The degradation kinetics of PEG-PCL-SNAP-tc grafts confirm acceptable stability of the grafts during the perceived healing period of 6 weeks.

\section{CONCLUSION}

In conclusion, this study describes a novel method to fabricate antibacterial and hemocompatible 3D-printed SDVGs. Results suggest that $3 \mathrm{D}$-printing is a promising method to produce SDVGs which can innovatively be combined with controlled NO release. These grafts were shown to successfully eradicate bacteria and inhibit platelet activation for a period lasting for 14 days. Considering these promising results, 3D printed NOreleasing grafts represent a promising design for tissue engineering of SDVGs, useful for future clinical applicability.

\section{ASSOCIATED CONTENT}

\section{S Supporting Information}

The Supporting Information is available free of charge on the ACS Publications website at DOI: 10.1021/acsbiomaterials.9b00220.

Weight of different coatings (Table S1), NO flux curve from different percentages of SNAP in PEG-PCL matrix (Figure S1), and the percentage of platelet aggregation in the presence of $48 \mathrm{~h}$ presoaked grafts (Figure S2) (PDF)

\section{AUTHOR INFORMATION}

\section{Corresponding Author}

*E-mail: mozafari.masoud@gmail.com. Phone/fax: 00989126490679.

\section{ORCID $\odot$}

\section{Masoud Mozafari: 0000-0002-0232-352X}

\section{Author Contributions}

F.K. collected and analyzed the data. F.K., B.D., M.M., A.Z., R.H., and M.F.H. designed this research. M.M., B.D., R.H., and M.F.H. supervised the project. F.K. drafted the manuscript. B.D., R.H., M.F.H., and M.M. edited the manuscript, and all authors read and approved the final manuscript.

\section{Notes}

The authors declare no competing financial interest.

\section{ACKNOWLEDGMENTS}

The authors would like to acknowledge Dr. Hamed Abedini for his technical assistance and fruitful discussion. We are grateful to Dr. Pieter Baatsen for kindly providing the SEM imaging. This study was supported by a grant of the Research Fund KU Leuven (Grant OT/14/097) given to R.H. and the Programmafinanciering KU Leuven (Grant PF/10/014). The Clinical Research Fund of UZ Leuven sponsored R.H.

\section{REFERENCES}

(1) Hasan, A.; Memic, A.; Annabi, N.; Hossain, M.; Paul, A.; Dokmeci, M. R.; Dehghani, F.; Khademhosseini, A. Electrospun scaffolds for tissue engineering of vascular grafts. Acta Biomater. 2014, 10 (1), 11-25.

(2) Xie, Y.; Guan, Y.; Kim, S.-H.; King, M. W. The mechanical performance of weft-knitted/electrospun bilayer small diameter vascular prostheses. I. Mech. Behav. Biomed. Mater. 2016, 61, 410418.

(3) Elomaa, L.; Yang, Y. P. Additive manufacturing of vascular grafts and vascularized tissue constructs. Tissue Eng., Part B 2017, 23 (5), 436-450.

(4) Gold, K.; Gaharwar, A. K.; Jain, A. Emerging trends in multiscale modeling of vascular pathophysiology: Organ-on-a-chip and 3D printing. Biomaterials 2019, 196, 2.

(5) Farzin, A.; Miri, A. K.; Sharifi, F.; Faramarzi, N.; Jaberi, A.; Mostafavi, A.; Solorzano, R.; Zhang, Y. S.; Annabi, N.; Khademhosseini, A. 3D-Printed Sugar-Based Stents Facilitating Vascular Anastomosis. Adv. Healthcare Mater. 2018, 7 (24), 1800702. (6) Melchiorri, A. J.; Hibino, N.; Best, C.; Yi, T.; Lee, Y.; Kraynak, C.; Kimerer, L. K.; Krieger, A.; Kim, P.; Breuer, C. K. 3D-Printed Biodegradable Polymeric Vascular Grafts. Adv. Healthcare Mater. 2016, 5 (3), 319-325.

(7) Touri, M.; Kabirian, F.; Saadati, M.; Ramakrishna, S.; Mozafari, M. Additive Manufacturing of Biomaterials- The Evolution of Rapid Prototyping. Adv. Eng. Mater. 2019, 21, 1800511.

(8) Wenger, R.; Giraud, M.-N. 3D Printing Applied to Tissue Engineered Vascular Grafts. Appl. Sci. 2018, 8 (12), 2631.

(9) Rosenzweig, D. H.; Carelli, E.; Steffen, T.; Jarzem, P.; Haglund, L. 3D-printed ABS and PLA scaffolds for cartilage and nucleus pulposus tissue regeneration. Int. I. Mol. Sci. 2015, 16 (7), 1511815135.

(10) Pavia, F. C.; La Carrubba, V.; Mannella, G. A.; Ghersi, G.; Brucato, V. Poly lactic acid based scaffolds for vascular tissue engineering. Chem. Eng. Trans. 2012, 27.

(11) Chia, H. N.; Wu, B. M. Recent advances in 3D printing of biomaterials. L. Biol. Eng. 2015, 9 (1), 4.

(12) Shalumon, K.; Deepthi, S.; Anupama, M.; Nair, S.; Jayakumar, R.; Chennazhi, K. Fabrication of poly (1-lactic acid)/gelatin composite tubular scaffolds for vascular tissue engineering. Int. J. Biol. Macromol. 2015, 72, 1048-1055.

(13) Kabirian, F.; Amoabediny, G.; Haghighipour, N.; Salehi-Nik, N.; Zandieh-Doulabi, B. Nitric oxide secretion by endothelial cells in response to fluid shear stress, aspirin, and temperature. J. Biomed. Mater. Res. Part A 2015, 103 (3), 1231-1237.

(14) Shalumon, K.; Chennazhi, K.; Nair, S. V.; Jayakumar, R. Development of small diameter fibrous vascular grafts with outer wall multiscale architecture to improve cell penetration. J. Biomed. Nanotechnol. 2013, 9 (7), 1299-1305.

(15) Charville, G. W.; Hetrick, E. M.; Geer, C. B.; Schoenfisch, M. H. Reduced bacterial adhesion to fibrinogen-coated substrates via nitric oxide release. Biomaterials 2008, 29 (30), 4039-4044.

(16) Wo, Y.; Brisbois, E. J.; Wu, J.; Li, Z.; Major, T. C.; Mohammed, A.; Wang, X.; Colletta, A.; Bull, J. L.; Matzger, A. J. Reduction of Thrombosis and Bacterial Infection via Controlled Nitric Oxide (NO) Release from S-Nitroso-N-acetylpenicillamine (SNAP) Impregnated Carbo Sil Intravascular Catheters. ACS Biomater. Sci. Eng. 2017, 3 (3), 349-359.

(17) Vermote, A.; Van Calenbergh, S. Small-molecule potentiators for conventional antibiotics against Staphylococcus aureus. ACS Infect. Dis. 2017, 3 (11), 780-796.

(18) Kim, S.; Choi, J. E.; Choi, J.; Chung, K.-H.; Park, K.; Yi, J.; Ryu, D.-Y. Oxidative stress-dependent toxicity of silver nanoparticles in human hepatoma cells. Toxicol. In Vitro 2009, 23 (6), 1076-1084.

(19) Brisbois, E. J.; Kim, M.; Wang, X.; Mohammed, A.; Major, T. C.; Wu, J.; Brownstein, J.; Xi, C.; Handa, H.; Bartlett, R. H. Improved Hemocompatibility of Multilumen Catheters Via Nitric Oxide (No) Release from S-Nitroso-N-Acetylpenicillamine (Snap) Composite Filled Lumen. ACS Appl. Mater. Interfaces 2016, 8 (43), 2927029279.

(20) Pant, J.; Goudie, M. J.; Hopkins, S. P.; Brisbois, E. J.; Handa, H. Tunable nitric oxide release from $\mathrm{S}$-nitroso- $\mathrm{N}$-acetylpenicillamine via catalytic copper nanoparticles for biomedical applications. ACS Appl. Mater. Interfaces 2017, 9 (18), 15254-15264.

(21) Wo, Y.; Brisbois, E. J.; Bartlett, R. H.; Meyerhoff, M. E. Recent advances in thromboresistant and antimicrobial polymers for 
biomedical applications: just say yes to nitric oxide (NO). Biomater. Sci. 2016, 4 (8), 1161-1183.

(22) Carpenter, A. W.; Schoenfisch, M. H. Nitric oxide release: Part II. Therapeutic applications. Chem. Soc. Rev. 2012, 41 (10), 37423752.

(23) Riccio, D. A.; Schoenfisch, M. H. Nitric oxide release: Part I. Macromolecular scaffolds. Chem. Soc. Rev. 2012, 41 (10), 3731-3741. (24) Schanuel, F. S.; Santos, K. S. R.; Monte-Alto-Costa, A.; de Oliveira, M. G. Combined nitric oxide-releasing poly (vinyl alcohol) film/F127 hydrogel for accelerating wound healing. Colloids Surf., B 2015, 130, 182-191.

(25) Brisbois, E. J.; Major, T. C.; Goudie, M. J.; Bartlett, R. H.; Meyerhoff, M. E.; Handa, H. Improved hemocompatibility of silicone rubber extracorporeal tubing via solvent swelling-impregnation of $S$ nitroso-N-acetylpenicillamine (SNAP) and evaluation in rabbit thrombogenicity model. Acta Biomater. 2016, 37, 111-119.

(26) Hansson, K. M.; Tosatti, S.; Isaksson, J.; Wetterö, J.; Textor, M.; Lindahl, T. L.; Tengvall, P. Whole blood coagulation on protein adsorption-resistant PEG and peptide functionalised PEG-coated titanium surfaces. Biomaterials 2005, 26 (8), 861-872.

(27) Repanas, A.; Wolkers, W.; Gryshkov, O.; Kalozoumis, P.; Mueller, M.; Zernetsch, H.; Korossis, S.; Glasmacher, B. Coaxial electrospinning as a process to engineer biodegradable polymeric scaffolds as drug delivery systems for anti-inflammatory and antithrombotic pharmaceutical agents. Clin. Exp. Pharmacol. 2015, 5, 192. (28) Mondal, D.; Griffith, M.; Venkatraman, S. S. Polycaprolactonebased biomaterials for tissue engineering and drug delivery: Current scenario and challenges. Int. I. Polvm. Mater. 2016, 65 (5), 255-265.

(29) Jiang, Y.; Mao, K.; Cai, X.; Lai, S.; Chen, X. Poly (ethyl glycol) assisting water sorption enhancement of poly $(\varepsilon$-caprolactone) blend for drug delivery. I. Appl. Polvm. Sci. 2011, 122 (4), 2309-2316.

(30) McCarthy, C. W.; Guillory, R. J.; Goldman, J.; Frost, M. C. Transition-metal-mediated release of nitric oxide (NO) from Snitroso-N-acetyl-d-penicillamine (SNAP): potential applications for endogenous release of $\mathrm{NO}$ at the surface of stents via corrosion products. ACS Appl. Mater. Interfaces 2016, 8 (16), 10128-10135.

(31) Miranda, K. M.; Espey, M. G.; Wink, D. A. A rapid, simple spectrophotometric method for simultaneous detection of nitrate and nitrite. Nitric Oxide 2001, 5 (1), 62-71.

(32) García-Robledo, E.; Corzo, A.; Papaspyrou, S. A fast and direct spectrophotometric method for the sequential determination of nitrate and nitrite at low concentrations in small volumes. Mar. Chem. 2014, 162, 30-36.

(33) Kabirian, F.; Ditkowski, B.; Zamanian, A.; Heying, R.; Mozafari, $\mathrm{M}$. An innovative approach towards 3D-printed scaffolds for the next generation of tissue-engineered vascular grafts. Mater. Today: Proc. 2018, 5 (7), 15586-15594.

(34) Claes, J.; Liesenborghs, L.; Peetermans, M.; Veloso, T.; Missiakas, D.; Schneewind, O.; Mancini, S.; Entenza, J.; Hoylaerts, M.; Heying, R. Clumping factor A, von Willebrand factor-binding protein and von Willebrand factor anchor Staphylococcus aureus to

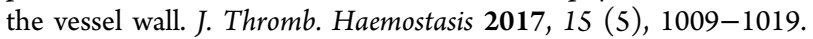

(35) Major, T. C.; Brisbois, E. J.; Jones, A. M.; Zanetti, M. E.; Annich, G. M.; Bartlett, R. H.; Handa, H. The effect of a polyurethane coating incorporating both a thrombin inhibitor and nitric oxide on hemocompatibility in extracorporeal circulation. Biomaterials 2014, 35 (26), 7271-7285.

(36) Wo, Y.; Li, Z.; Brisbois, E. J.; Colletta, A.; Wu, J.; Major, T. C.; Xi, C.; Bartlett, R. H.; Matzger, A. J.; Meyerhoff, M. E. Origin of longterm storage stability and nitric oxide release behavior of CarboSil polymer doped with S-nitroso-N-acetyl-D-penicillamine. ACS Appl. Mater. Interfaces 2015, 7 (40), 22218-22227.

(37) de Mel, A.; Murad, F.; Seifalian, A. M. Nitric oxide: a guardian for vascular grafts? Chem. Rev. 2011, 111 (9), 5742-5767.

(38) Tham, A. Y.; Gandhimathi, C.; Praveena, J.; Venugopal, J. R.; Ramakrishna, S.; Kumar, S. D. Minocycline Loaded Hybrid Composites Nanoparticles for Mesenchymal Stem Cells Differentiation into Osteogenesis. Int. I. Mol. Sci. 2016, 17 (8), 1222.
(39) Pavia, F. C.; La Carrubba, V.; Piccarolo, S.; Brucato, V. Polymeric scaffolds prepared via thermally induced phase separation: tuning of structure and morphology. J. Biomed. Mater. Res., Part A 2008, 86 (2), 459-466.

(40) Ovsianikov, A.; Schlie, S.; Ngezahayo, A.; Haverich, A.; Chichkov, B. N. Two-photon polymerization technique for microfabrication of CAD-designed 3D scaffolds from commercially available photosensitive materials. J. Tissue Eng. Regener. Med. 2007, 1 (6), 443-449.

(41) Baudis, S.; Nehl, F.; Ligon, S. C.; Nigisch, A.; Bergmeister, H.; Bernhard, D.; Stampfl, J.; Liska, R. Elastomeric degradable biomaterials by photopolymerization-based CAD-CAM for vascular tissue engineering. Biomed. Mater. 2011, 6 (5), 055003.

(42) Meyer, W.; Engelhardt, S.; Novosel, E.; Elling, B.; Wegener, M.; Krüger, H. Soft polymers for building up small and smallest blood supplying systems by stereolithography. J. Funct. Biomater. 2012, 3 (2), 257-268.

(43) Liu, Y.; Xiang, K.; Chen, H.; Li, Y.; Hu, Q. Composite vascular repair grafts via micro-imprinting and electrospinning. AIP Adv. 2015, 5 (4), 041318.

(44) Melchiorri, A. J.; Hibino, N.; Best, C.; Yi, T.; Lee, Y.; Kraynak, C.; Kimerer, L. K.; Krieger, A.; Kim, P.; Breuer, C. K. 3D-Printed Biodegradable Polymeric Vascular Grafts. Adv. Healthcare Mater. 2016, 5 (3), 319-325.

(45) Fukunishi, T.; Best, C. A.; Sugiura, T.; Opfermann, J.; Ong, C. S.; Shinoka, T.; Breuer, C. K.; Krieger, A.; Johnson, J.; Hibino, N. Preclinical study of patient-specific cell-free nanofiber tissueengineered vascular grafts using 3-dimensional printing in a sheep model. I. Thorac. Cardiovasc. Surg. 2017, 153 (4), 924-932.

(46) Brisbois, E. J.; Davis, R. P.; Jones, A. M.; Major, T. C.; Bartlett, R. H.; Meyerhoff, M. E.; Handa, H. Reduction in thrombosis and bacterial adhesion with 7 day implantation of S-nitroso-Nacetylpenicillamine (SNAP)-doped Elast-eon E2As catheters in sheep. I. Mater. Chem. B 2015, 3 (8), 1639-1645.

(47) Wo, Y.; Li, Z.; Colletta, A.; Wu, J.; Xi, C.; Matzger, A. J.; Brisbois, E. J.; Bartlett, R. H.; Meyerhoff, M. E. Study of crystal formation and nitric oxide (NO) release mechanism from S-nitroso$\mathrm{N}$-acetylpenicillamine (SNAP)-doped CarboSil polymer composites for potential antimicrobial applications. Composites, Part B 2017, 121, $23-33$.

(48) Chen, C.-H.; Chen, S.-H.; Shalumon, K.; Chen, J.-P. Prevention of peritendinous adhesions with electrospun polyethylene glycol/ polycaprolactonenanofibrous membranes. Colloids Surf., B 2015, 133, 221-230.

(49) Chen, M.-H.; Liang, P.-C.; Chang, K.-C.; Huang, J.-Y.; Chang, Y.-T.; Chang, F.-Y.; Wong, J.-M.; Lin, F.-H. Prototype of biliary drugeluting stent with photodynamic and chemotherapy using electrospinning. Biomed. Eng. online. 2014, 13 (1), 118

(50) Seager, R.; Acevedo, A. J.; Spill, F.; Zaman, M. H. Solid dissolution in a fluid solvent is characterized by the interplay of surface area-dependent diffusion and physical fragmentation. Sci. Rep. 2018, 8, 8 .

(51) Brisbois, E. J.; Handa, H.; Major, T. C.; Bartlett, R. H.; Meyerhoff, M. E. Long-term nitric oxide release and elevated temperature stability with S-nitroso-N-acetylpenicillamine (SNAP)doped Elast-eon E2As polymer. Biomaterials 2013, 34 (28), 69576966.

(52) Azarov, I.; He, X.; Jeffers, A.; Basu, S.; Ucer, B.; Hantgan, R. R.; Levy, A.; Kim-Shapiro, D. B. Rate of nitric oxide scavenging by hemoglobin bound to haptoglobin. Nitric Oxide 2008, 18 (4), 296302.

(53) Behzadi, S.; Serpooshan, V.; Sakhtianchi, R.; Müller, B.; Landfester, K.; Crespy, D.; Mahmoudi, M. Protein corona change the drug release profile of nanocarriers: the "overlooked" factor at the nanobio interface. Colloids Surf. B 2014, 123, 143-149.

(54) Pant, J.; Gao, J.; Goudie, M. J.; Hopkins, S. P.; Locklin, J.; Handa, H. A multi-defense strategy: Enhancing bactericidal activity of a medical grade polymer with a nitric oxide donor and surface- 
immobilized quaternary ammonium compound. Acta Biomater. 2017, $58,421-431$.

(55) Lee, W. H.; Ren, H.; Wu, J.; Novak, O.; Brown, R. B.; Xi, C.; Meyerhoff, M. E. Electrochemically Modulated Nitric Oxide Release From Flexible Silicone Rubber Patch: Antimicrobial Activity For Potential Wound Healing Applications. ACS Biomater. Sci. Eng. 2016, 2 (9), 1432-1435.

(56) Mihu, M. R.; Cabral, V.; Pattabhi, R.; Tar, M. T.; Davies, K. P.; Friedman, A. J.; Martinez, L. R.; Nosanchuk, J. D. Sustained nitric oxide-releasing nanoparticles interfere with methicillin-resistant Staphylococcus aureus adhesion and biofilm formation in a rat central venous catheter model. Antimicrob. Agents Chemother. 2017, AAC, 02020-16.

(57) Seabra, A. B.; Martins, D.; Simões, M. M.; Da Silva, R.; Brocchi, M.; De Oliveira, M. G. Antibacterial nitric oxide-releasing polyester for the coating of blood-contacting artificial materials. Artif. Organs 2010, 34 (7), E204-E214.

(58) Mai-Prochnow, A.; Clauson, M.; Hong, J.; Murphy, A. B. Gram positive and Gram negative bacteria differ in their sensitivity to cold plasma. Sci. Rep. 2016, 6, 38610.

(59) Silhavy, T. J.; Kahne, D.; Walker, S. The bacterial cell envelope. Cold Spring Harbor Perspect. Biol. 2010, 2, No. a000414.

(60) Major, T. C.; Brant, D. O.; Reynolds, M. M.; Bartlett, R. H.; Meyerhoff, M. E.; Handa, H.; Annich, G. M. The attenuation of platelet and monocyte activation in a rabbit model of extracorporeal circulation by a nitric oxide releasing polymer. Biomaterials 2010, 31 (10), 2736-2745.

(61) Goudie, M. J.; Pant, J.; Handa, H. Liquid-infused nitric oxidereleasing (LINORel) silicone for decreased fouling, thrombosis, and infection of medical devices. Sci. Rep. 2017, 7 (1), 13623.

(62) Radomski, M. W.; Rees, D. D.; Dutra, A.; Moncada, S. Snitroso-glutathione inhibits platelet activation in vitro and in vivo. $B r$. J. Pharmacol. 1992, 107 (3), 745-749.

(63) Yang, Z.; Yang, Y.; Xiong, K.; Li, X.; Qi, P.; Tu, Q.; Jing, F.; Weng, Y.; Wang, J.; Huang, N. Nitric oxide producing coating mimicking endothelium function for multifunctional vascular stents. Biomaterials 2015, 63, 80-92.

(64) Gimeno, M.; Pinczowski, P.; Pérez, M.; Giorello, A.; Martínez, M. A.; Santamaría, J.; Arruebo, M.; Luján, L. A controlled antibiotic release system to prevent orthopedic-implant associated infections: An in vitro study. Eur. I. Pharm. Biopharm. 2015, 96, 264-271.

(65) Xu, L.-C.; Wo, Y.; Meyerhoff, M. E.; Siedlecki, C. A. Inhibition of bacterial adhesion and biofilm formation by dual functional textured and nitric oxide releasing surfaces. Acta Biomater. 2017, 51, $53-65$.

(66) Barbato, J. E.; Tzeng, E. Nitric oxide and arterial disease. J. Vasc. Surg. 2004, 40 (1), 187-193.

(67) Melchiorri, A. J.; Hibino, N.; Fisher, J. P. Strategies and techniques to enhance the in situ endothelialization of small-diameter biodegradable polymeric vascular grafts. Tissue Eng., Part B 2013, 19 (4), 292-307. 